JINYU JIANG, Ph.D. Candidate

E-mail: jiangjy@dlmu.edu.cn

XU ZHAO, Ph.D. ${ }^{1}$

E-mail: zhao_xu@126.com

WEIYOU GUO, Ph.D. Candidate ${ }^{1}$

E-mail: 18840839938@163.com

ZHONGZHEN YANG, Ph.D. ${ }^{1,2}$

(Corresponding author)

E-mail: yangzhongzhen@nbu.edu.cn

${ }^{1}$ College of Transport Engineering

Dalian Maritime University

No. 1 Linghai Road, Dalian, 116026, China

${ }^{2}$ Faculty of Maritime and Transportation

Ningbo University

No. 818 Fenghua Road, Ningbo, 315832, China
Traffic on Motorways Original Scientific Paper Submitted: 6 Nov. 2019 Accepted: 16 July 2020

\title{
SCHEME OF OVERLOADED TRUCK CONTROL ON A RURAL HIGHWAY
}

\begin{abstract}
A new working mode of overloaded traffic control for rural highways is presented, and a location-routing model is built to optimize the check base distribution and the control vehicles' routing schemes. Then, for the location-routing model with a large set of location alternatives and an unknown settable number of check bases, a multiple ant colony optimization algorithm is designed to solve the model. Furthermore, actual data from Guiyang rural highways are used to perform a numerical analysis. The results indicate that the model can be used to obtain the optimal base location-vehicle routing scheme to verify the feasibility of the model and the algorithm. The model and algorithm can help managers to make decisions on locating the check bases and routing the control vehicles.
\end{abstract}

\section{KEYWORDS:}

highway traffic; location and routing model; multiple ant colony optimization algorithm; check base; rural highway;

\section{INTRODUCTION}

The issue of truck overloading can commonly be seen in China. Overloaded trucks damage road pavement and shorten road service life [1]. Therefore, China has been intensifying control over overloaded trucks on highways. Since 2004 China has continuously implemented controls for overloaded trucks [2]. On rural highways, the common way to control overloaded trucks is to set the monitoring stations (hereafter check bases) along the roadways to check the passing traffic in order to find and penalize the overloaded trucks.

However, it is difficult to effectively intercept the overloaded trucks with this method because rural road networks are too connected and complicated, and the overloaded trucks may detour around the fixed check bases at little cost. To improve the efficiency and effect of overload control, this paper puts forward a new working mode for overload control, namely, setting some check bases first and then dispatching control vehicles from the bases to capture the overloaded trucks by cruising nearby rural highways. In contrast to fixed check points, the control vehicles can visit all roadways to overawe all the overloaded trucks. However, it cannot be expected that the control vehicle can capture all overloaded trucks in the rural highway network. Apart from expressways with on-off ramps, in which all overloaded trucks can be found at the toll gates, for other open roads (such as normal highway or rural road) it is impossible to catch all overloaded trucks. The point of this study is not to find all overloaded trucks, but to make control vehicles visiting all overloaded roadways at the minimum cost and to make all overloaded trucks afraid of being captured. The idea of using control vehicles to capture the overloaded trucks has been put forward based on a World Bank-funded project in Guiyang. The feasibility and efficiency have been discussed soundly and some trial projects are being discussed. This study intends to optimize the routes of control vehicles and 
the distribution of check bases in the case that the advantage of the input-output of new mode has been commonly realized by the authorities.

Currently, not much literature exists on controlling overloaded trucks. Bagui provided a definition of an overloaded truck and proposed a method to compensate for road damages by fining the overloaded trucks [3]. Quintero et al. proposed a bi-level model to represent the interactions between the truck loading practices of freight transport carriers and the decisions of a road planning authority which is responsible for the enforcement of the overloading control [4]. The model can predict a carrier's reactions under a series of planner's decisions and then help the planner choose an optimal combination of the number of check points and the punishment levels to have the minimal total expenditure (on repairs and overloading inspection costs). However, the issues regarding the base location and control vehicle routing have not been addressed in the study. $\mathrm{Li}$ analysed the interactions between a carrier's profit, transport price, operating cost and overloading penalties, and gave the solutions and the policy implications for China to deal with the overloading issue [5]. Chen presented the idea of tolling the trucks by actual loaded weights and axle loading quotas and studied the long-term effectiveness of controlling the overloaded trucks by economic methods [6]. The above review shows that, except for Quintero's study on the number of check bases and checking the frequency of overloaded trucks, all the other literature studied the governance of overloading at the macro level, such as analyses of policies and strategies for overloaded traffic control, and lacked specific quantitative analyses for the control of the overloaded trucks (including the location of check bases, the routing of control vehicles, etc.).

Salhi proposed the Location-Routing Problem (LRP) which is a generalization of the Vehicle-Routing Problem (VRP) that reflects the interdependence between facility locations and vehicle-routing decisions [7]. Nagy defined LRP as an operation issue to minimize the total cost of construction and transport by determining the locations of facilities and the vehicle travel routes when the location alternatives and client sites are given [8]. In recent years, the LRP research community has been very active. The location-routing model is widely used in many fields. For example, under the constraints of facility capacity and vehicle capacity, Luo established a two-objective LRP model with a fuzzy time window based on the rule of minimizing the total cost and maximizing the customer satisfaction [9]. Taking into consideration the demand changes of customers, Nadizadeh established a dynamic location-routing optimization model in the case of limited capacities of vehicles and warehouses [10]. More studies on LRP can be found in literature [11-15].

Although the location-routing model has been widely used on the location of distribution centres, warehouses, and emergency facilities, it is still in the early trial stage for the microlevel decisions on the spatial distribution of check bases and the number of control vehicles in each base and the corresponding cruise routes.

Several exact algorithms have been devoted to solving the LRP. Laporte and Norbert designed a branch-and-bound algorithm with a single depot and solved instances that had 20-50 customers and 2-5 routes per opened depot [16]. For a given number of customers, LRPs tend to be more difficult to solve when the number of depots gets larger. Then, to solve the multi-depot location-routing problems, Laporte et al. designed a branch-and-bound algorithm which can solve LRPs with up to 2-3 depots and 40 customers [17]. Akca et al. presented a capacitated location and routing problem (CLRP) and designed a branch-and-price algorithm with up to 40 customers [18]. The size of the LRP is limited when the LRP is solved by exact algorithms. Srivastava et al. proposed such approaches may work only for medium-sized location-routing problems [19]. Furthermore, when time window and/or route distance constraints are added, the problems become even harder to solve.

As the LRP problem is an NP-hard problem, most of the research studies used a heuristics algorithm for the solution. In literature [8], Nagy and Salhi classified the heuristics algorithm into four types as follows: sequential, clustering-based, iterative, and hierarchical. Sequential methods first optimize the depot location and then optimize the vehicle routes based on the located depots [20]. There is no feedback between location optimization and vehicle routing. Balakrishnan et al., Salhi and Rand, and Salhi and Nagy pointed out that the sequential solution for VRP cannot guarantee the optimal scheme for the distribution system [7, 21, 22]. However, Srivastava and Benton found that sequential methods can provide good quality solutions in some cases [23]. Sérgio Barreto et al. presented a cluster analysis based on a sequential 
heuristics [24]. They constructed groups of customers with the capacity limitation and determined the distribution route in each customer group first and then located the distribution centres and assigned the routes to them. Wu et al. decomposed the LRP into two sub-problems (location-allocation problem, vehicle-routing problem) first and then solved the sub-problems iteratively by feeding information from one sub-problem to the other [25]. Hierarchical methods (Salhi et al.; Nambiar et al.) consider the routing problem to be a subordinated problem that is nested into the main location problem and solve the LRP as a whole [26, 27].

According to the existing literature, the problem scale is generally 2-200 nodes (including location alternatives and demand points). The literature on large-sized LRPs focuses on the LRP with a large number of demand points, while the scale of the location alternatives is small (we can see this point in literature $[9-12,14,15])$. Therefore, the solution of these LRPs can be obtained based on the combination of a simple heuristic algorithm and an enumeration method. However, when the set scale of location alternatives gets larger and the maximum number of facilities is not given, a combinatorial explosion will occur of the number of potential location combinations, which leads to the solution space increasing sharply. It is not realistic to use the enumeration method to solve the vehicle cruising scheme of location combinations one by one. Therefore, some researchers proposed the heuristic approach that hybrid multiple heuristics approaches to solve the LRP. In 2005, Albareda-Sambola et al. proposed a heuristic algorithm combining taboo heuristic algorithm and rounding heuristic algorithm to solve the LRP [28]. In 2006, Bouhafs et al. proposed a combination of simulated annealing and ant colony system to solve the capacitated LRP [29]. In 2008, Marinakis and Marinaki proposed a bi-level genetic algorithm to solve the LRP [30]. Inspired by their studies, this paper attempts to use the heuristic that hybrid multiple heuristic approaches to solve LRP in the case of a large set of alternatives.

Under the working mode of a mobile overload control for rural highways by control vehicles based on check bases, this paper first constructs an optimization model that can simultaneously determine the number and location of check bases and the cruise routes of control vehicles, with the aim of visiting all the roadways with overloaded trucks for the minimum cost. Then, a multiple ant colony optimization algorithm is designed to solve this LRP with a large set scale of location alternatives. Finally, the actual data on Guiyang rural highways are used to conduct a numerical test. The results show that by the optimal location-routing scheme, check bases are selected in areas with abundant mineral resources and dense construction sites, where large trucks such as mineral trucks and slag trucks are often overloaded. The designed routes of the control vehicles consist of many provincial roadways and county-level and township-level rural roadways. These roadways have heavy truck traffic. These cruise routes can ensure that the control vehicles effectively intercept the overloaded trucks. It will be shown that our method is helpful for implementing the bases' spatial distribution decisions and vehicle cruise route schemes.

The remainder of this paper is arranged as follows. In Section 1, a programming model is constructed that simultaneously locates the check bases and assigns routes for the control vehicles and clears the goal that all roadways with overloaded trucks are visited with the minimum cost. In Section 2, an algorithm is designed to solve the model. In Section 3 , a numerical analysis is done with the actual data on Guiyang rural highways. Finally, in Section 4, the whole study has been summarized.

\section{LOCATION-ROUTING MODEL}

As shown in Figure 1, roadways with overloaded trucks in a road network are mapped into discrete points to form a set of control demand points. To control the overloaded trucks, each check base should be equipped with at least one control vehicle, and all vehicles are of the same type. A control vehicle, which is equipped with mobile weighing facilities, starts cruising from a check base to visit the control demand points to detect the overloaded trucks. In

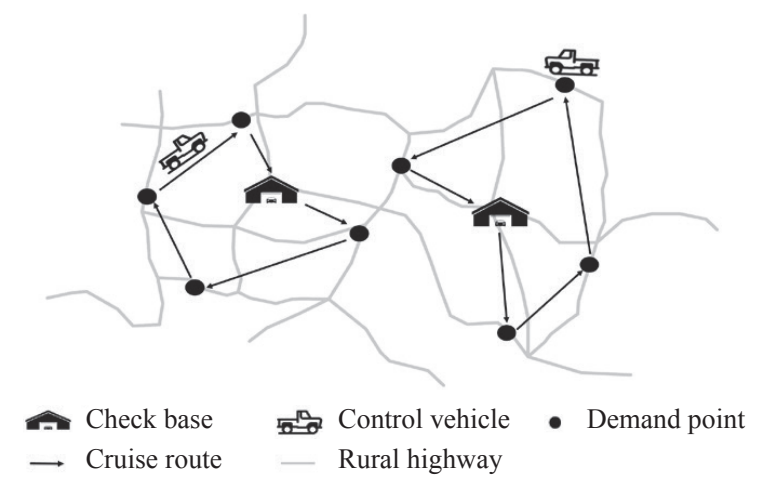

Figure 1 - Schematic diagram of overload control by mobile facilities 
its cruising trip, a control vehicle will stop at some well-sighted sites along a roadway to set a temporal check point. The administrative staff guide the suspected trucks which pass along the roadway to the check point and weight them by mobile weighing equipment.

The staying and working of a control vehicle at each demand point is the same, and a demand point is visited only once. The travel time of a control vehicle for a cruising loop should not be longer than the vehicle maximum travel range. Every control vehicle should return to the base from which it has departed.

To monitor all of the control demand points, the problem of selecting a number of check bases from a set of alternatives and assigning some control vehicle to each check base, as well as designing the control vehicle cruise network is studied in the context of a known road network and a set of control demand points. The goal of the decision-making is to maximize the control efficiency with as little control cost as possible, which consists of a set of sub-costs, including the cost for constructing check bases, the cost of purchasing control vehicles, the tour cost of control vehicles, and the labour cost.

\section{Variables and parameters}

$J$ - set of location alternatives of check bases;

$I$ - set of control demand points;

$K$ - set of control vehicles;

$c_{j}$ - fixed cost of a single check base;

$c_{k}$ - fixed cost of one control vehicle;

$c_{l}$ - unit transport cost;

$c_{p}$ - unit labour cost;

$v$ - travel speed for control vehicle;

$t$ - staying and working time of a control vehicle at a demand point;

$t_{\max }$ - maximum daily travel time of a control vehicle;

$l_{i i}$, - distance between two demand points;

$n$ - number of attached staffs for one control vehicle;

$u$ - maximum equipped number for control vehicle;

$D_{k}$ - number of demand points visited by control vehicle $k$;

$z_{j}=1$ if a check base is located at point $j$, 0 otherwise;

$z_{j k}=1$ if check base $j$ selects control vehicle $k$, 0 otherwise;

$x_{i i^{\prime} k}=1$ if control vehicle $k$ travels from demand point $i$ to demand point $i$ ', 0 otherwise.

\section{Model formula}

$$
\begin{aligned}
& \operatorname{Min} C=\sum_{j \in J} \sum_{k \in K} \sum_{i, i \in I \cup J} z_{j} c_{j}+z_{j k} c_{k}+x_{i i k}{ }^{\prime} l_{i i}{ }^{\prime} c_{l}+n \cdot z_{j k} c_{p} \\
& \text { s.t. } z_{j} \in(0,1), j \in J \\
& z_{j k} \in(0,1), k \in K \\
& x_{i j k} \in(0,1), \quad i \in I \cup J, \quad j \in I \cup J, k \in K \\
& \sum_{i \in I \cup J} x_{i j k}-\sum_{i \in I \cup J} x_{j i k}=0, \quad j \in I, k \in K \\
& \sum_{k \in K} \sum_{i \in I \cup J} x_{i j k}=1, \quad j \in I \\
& \sum_{i \in I} x_{j i k}=\sum_{i \in I} x_{i j k}, \quad j \in J, k \in K \\
& z_{j} \geq \sum_{i \in I} x_{j i k}, k \in K, \quad j \in J \\
& \sum_{i, j \in I} x_{i j k} \cdot \frac{l_{i j}}{v}+D_{k} \cdot t \leq t_{\max }, k \in K \\
& \sum_{j \in J} \sum_{k \in K} z_{j k} \leq u
\end{aligned}
$$

where: $C$ is the total cost for controlling overloaded trucks, including the fixed cost of base construction, the fixed cost of control vehicle purchase, the fuel cost and the labour cost. Equation 1 is the objective function denoting the minimization of the total cost. Equation 5 ensures that the cruise route of a control vehicle is a closed loop. Equation 6 ensures that a demand point can only be served by one vehicle. Equation 7 ensures that the control vehicle starts from a check base and eventually returns back to the same base. Equation 8 indicates that as long as a control vehicle starts from a location alternative, the base is set at that alternative. Equation 9 ensures that the cruise time of each control vehicle is shorter than its daily cruise limitation. Equation 10 illustrates that the number of control vehicles is smaller than the maximum number.

\section{ALGORITHM}

The above problem is a non-linear programming problem that is composed of a Facility Location Problem (FLP) and a vehicle-routing problem, namely, a location-routing problem. It is an NP-hard problem and usually needs to be solved by a heuristic algorithm [31]. In literature [8], Nagy and Salhi believed that using hierarchical heuristic algorithms to solve LRPs may provide better solutions. Based on their research, the proposed Multiple Ant Colony Optimization algorithm (MACO) in this paper is hierarchical. 
The Ant Colony Optimization algorithm (ACO) was first proposed by Dorigo et al. [32]. Then the ACO has been improved by some researchers [33, 34]. However, MACO was first proposed by Gambardella for solving the VRP with time windows [35]. They designed two ant colonies to optimize two different objective functions. Ting et al. adopted the MACO to solve the LRP with capacity constraints on depots and routes [36]. They decomposed the LRP into a facility location problem and a multiple depot vehicle-routing problem, and, as the result, the vehicle-routing problem is nested into the facility location problem. In the research done by Ting et al., each ant of the FLP may generate a different number of locations. This means that the number of facilities selected by an ant is random, but through the accumulation of pheromones on the ants' routes, the algorithm can obtain the final location scheme by an iterative calculation. In this paper, in order to avoid local optimal solution caused by the randomly generated facility number, all the possible numbers of facilities are enumerated, and the location-routing problem is correspondingly solved. When the algorithm termination condition is met, the calculation ends and the global optimal solution is output. The specific method is that MACO is adopted to solve the LRP from the case of one check base (namely, $H=1$ ) to $H=H_{\max }$ check bases $\left(H_{\max }\right.$ is the number of alternative locations). For each $H$, there is an optimal location-route scheme (a local optimal solution for the whole LRP). Finally, the global optimal solution can be found from these local optimal solutions. In the process of calculation, the optimal location-routing scheme for each $H$ need not be obtained. $C_{H}$ is assumed to be the total cost for overloaded traffic control in the optimal location-route scheme with $H$ check bases being set. $c_{H}$ is the fixed cost which is proportional to the number of bases. When $c_{H+1}>\operatorname{Min}\left(C_{H}, C_{H-1}, \ldots C_{1}\right)$, it means that the lowest cost for the overloaded traffic control is $\operatorname{Min}\left(C_{H}, C_{H-1}, \ldots C_{1}\right)$, namely, the global optimal solution has been determined and $H$ need not be increased anymore to solve the LRP.

The algorithm structure is shown in Figure 2. A hierarchical ACO structure is designed with different transition rules for the upper-level ant colony and the lower-level ant colony. The upper-level is for the location decision, while the lower-level is for the route selection. The route selection is nested into the location decision process. Namely, for the base(s) selected by each ant in the upper level, a route selection is carried out by the ant colony algorithm in the lower level. The coordination between the upper and lower levels is achieved by the global pheromone updating rule of the upper-level ant colony (the specific description can be seen in Section 2.4).

\subsection{Location selection}

When $H$ check bases are open, each ant in the upper level may generate a route. The nodes on each route are regarded as a location combination. This means that each ant in the upper level may generate a location combination. In the process of location

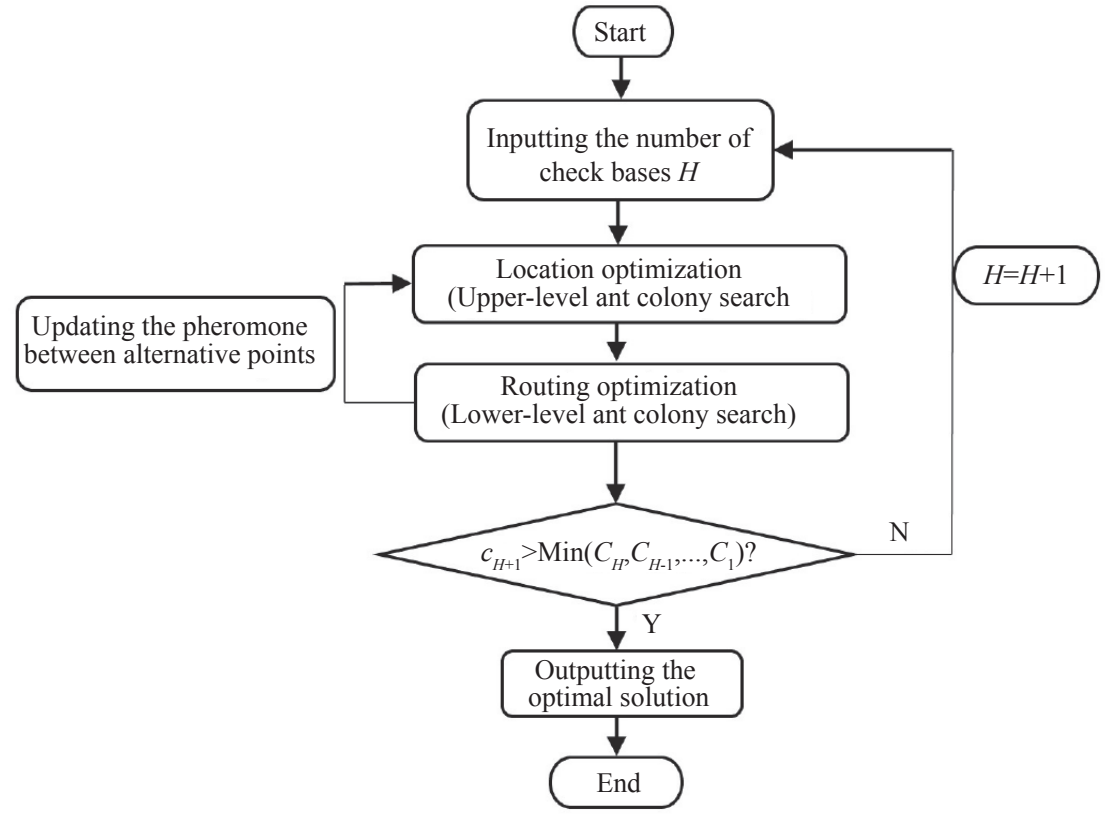

Figure 2 - The process diagram of the multiple ant colony algorithm 
selection, the probability of ants selecting every feasible node is determined by the selection expectation and pheromone between the current node and the feasible node. The specific calculation method of the selection probability is as follows:

First, based on the maximum cruise time of the vehicles, the set of demand points that can be covered by each alternative point is calculated and defined as the coverage range. The similarity is used to represent the size of the overlapping coverage range between the alternative points. The calculation method is shown in Equation 11, and then the selection expectation is defined as the reciprocal of the similarity between the alternative points (Equation 12).

$S_{a b}=\frac{L_{a b}}{L_{a}}, a \neq b, \quad b=1,2,3, \ldots, M$

Here, $S_{a b}$ is the similarity between the current alternative point $a$ and another alternative point $b ; L_{a b}$ is the number of demand points checked repeatedly by the current alternative point $a$ and the other alternative point $b ; L_{a}$ is the number of demand points checked by the current alternative point $a$.

$\eta_{a b}=\frac{1}{S_{a b}}$

where: $\eta_{a b}$ is the selection expectation of alternative point $b$ at alternative point $a$. $S_{a b}$ is the similarity between alternative point $a$ and alternative point $b$.

All ants select the next node from the starting point at the same time, and the departure time is set to 0 . When all ants complete a state transition (transferring from the current node to the next node), the time increases by 1 . The probability that an ant selects the alternative point $b$ from the alternative $a$ at any time $t$ is:

$$
p_{a b}(t)=\frac{\tau_{a b}^{\alpha}(t) \cdot\left(\eta_{a b}\right)^{\beta}}{\sum_{s \in S} \tau_{a s}^{\alpha}(t) \cdot\left(\eta_{a s}\right)^{\beta}}
$$

where: $\tau_{a b}(t)$ is the pheromone concentration of edge $(a, b)$ at time $t ; \eta_{a b}$ is the expectation of the edge $(a, b) ; \alpha$ and $\beta$ are the heuristic factors of pheromone concentration and expectation, respectively; and $S$ is a set of feasible alternative points.

\subsection{Vehicle routing}

In most of the literature on VRP, the customer assignment is required before the vehicle routing. Unlike most literature, here the demand points are not assigned to each base directly. Instead, for each location combination output by an upper ant, the check bases are mapped to a virtual check base and it is assumed that all the ants of the VRP take the virtual base as their starting point. The distance between the demand point $i$ and the virtual base is represented by the distance from the demand point $i$ to the nearest check base $d_{i}$. This method of changing the multiple depots into a single depot by using a virtual depot has been adopted in MA et al. [37]. In the process of vehicle routing, first, we set the maximum time that an ant can travel equal to the maximum time that a vehicle can cruise. Next, let the ants leave the virtual base to visit the demand points. The first demand point selected by the ant after its departure is defined as $i$, and the nearest real base to $i$ is $o_{i}$. The ant must visit $o_{i}$ after visiting several demand points (namely, $o_{i}$ must be added to the route), and the ant's travel time from the virtual base to $o_{i}$ must be shorter than the maximum time that an ant can travel. To ensure the ant can visit all the demand points, we set the ant's travel time equal to 0 when the ant arrives at $o_{i}$. Meanwhile, set the ant to select the next demand point from the virtual base rather than from $o_{i}$. Then, with the above steps, let the ant continue to visit the remaining demand points until all the demand points are visited.

For each single VRP base, the vehicle routing is carried out. In the process of routing, let $\eta_{i j}=1 / l_{i j}$ be the selection expectation of edge $(i, j)$, then, the probability that the ant starting from node $i$ selects node $j$ at time $t$ is:

$$
p_{i j}(t)=\frac{\tau_{i j}^{\alpha}(t) \cdot\left(\eta_{i j}\right)^{\beta}}{\sum_{s \in S} \tau_{i s}^{\alpha}(t) \cdot\left(\eta_{i j}\right)^{\beta}}
$$

where: $\tau_{i j}(t)$ is the pheromone concentration of edge $(i, j)$ at time $t ; \eta_{i j}$ is the expectation of edge $(i, j) ; \alpha$ and $\beta$ are the heuristic factors of the pheromone concentration and expectation, respectively; and $S$ is a set of feasible nodes.

\subsection{Pheromone updating rules of VRP}

There are two ways to update the pheromones between nodes [38]. One is the local pheromone updating. That is, after the ant walks through the edge between two nodes, the pheromone on the edge is updated. The updating rule is shown in Equations 15 and 16. Updating a local pheromone with negative feedback rules can reduce the possibility that subsequent ants choose the same edge, avoid premature stagnation of the algorithm, and improve the ants' global searching ability.

$$
\tau_{i j}(t+1)=\left(1-\rho_{1}\right) \tau_{i j}(t)+\rho_{1} \tau_{0}
$$




$$
\tau_{0}=\frac{1}{N \cdot C^{N N}}
$$

where: $\tau_{i j}(t)$ is the pheromone of edge $(i, j)$ at time $t$; $\alpha_{1}$ is the local pheromone updating parameter in the route optimization; $\rho_{1} \in(0,1) ; \tau_{0}$ is the initial value of the pheromone matrix; $N$ is the number of nodes; and $C^{N N}$ is the length of routes constructed by the nearest neighbourhood heuristics.

The other updating method is global pheromone updating, which is based on the elitist ant strategy in the ant system. Only one ant (the best-so-far ant of the VRP) is allowed to add a pheromone after each iteration of the calculation. The updating rule is shown in Equations 17 and 18. At this moment, the result of the best-so-far ant is converted into a pheromone increment to form positive feedback, which can accelerate the convergence speed of the algorithm.

$$
\begin{aligned}
\tau_{i j}^{T C+1} & =\left(1-\rho_{2}\right) \tau_{i j}^{T C}+\rho_{2} \Delta \tau_{i j}^{b s} \\
\Delta \tau_{i j}^{b s} & =\frac{1}{C_{L C}^{b s}}
\end{aligned}
$$

where: $\tau_{i j}^{T C}$ is the pheromone on edge $(i, j)$ after the $T C$-th iteration; $\rho_{2}$ is the global pheromone evaporation coefficient in route optimization, $\rho_{2} \in(0,1) ; \Delta \tau_{i j}^{b s}$ is the pheromone released by the best-so-far ant of the VRP on edge $(i, j)$ in the $T C$-th iteration; and $C_{L C}^{b s}$ is the cost of the control vehicles corresponding to the route of the best-so-far ant of the VRP under a location combination (including fixed vehicle cost, fuel cost, labour cost).

\subsection{Pheromone updating rules of FLP}

Similar to the pheromone updating rules of the VRP, there are two ways to update the pheromones. The local pheromone updating is carried out after each ant in the upper level selects the alternative point $b$ from alternative $a$. The updating rule is as follows:

$$
\begin{aligned}
& \tau_{a b}(t+1)=\left(1-\rho_{3}\right) \tau_{a b}(t)+\rho_{3} \tau_{a b}^{0} \\
& \tau_{a b}^{0}=\frac{1}{S_{a b}}
\end{aligned}
$$

where: $\tau_{a b}(t)$ is the pheromone on edge $(a, b)$ at time $t ; \rho_{3}$ is the local pheromone update parameter in the location optimization, $\rho_{3} \in(0,1)$; $\tau_{a b}$ is the initial value of the pheromone matrix; and $S_{a b}$ is the similarity between alternative points $a$ and $b$.

After the vehicle routing for all location combinations is selected by the upper-level ants in each iteration of the calculation, the best-so-far ant of the
FLP can be obtained, and only the best-so-far ant of the FLP is allowed to add a pheromone on the edge after each iteration of the FLP. The updating rule is as follows:

$$
\begin{aligned}
& \tau_{a b}^{T C+1}=\left(1-\rho_{4}\right) \tau_{a b}^{T C}+\rho_{4} \Delta \tau_{a b}^{b s} \\
& \Delta \tau_{a b}^{b s}=\frac{1}{C_{H}^{b s}}
\end{aligned}
$$

where: $\tau_{a b}^{T C}$ is the pheromone on the edge $(a, b)$ after the $T C$-th iteration; $\rho_{4}$ is the global pheromone evaporation coefficient in the location optimization, $\rho_{4} \in(0,1) ; \Delta \tau_{a b}^{b s}$ represents the pheromone released by the best-so-far ant of the FLP on the edge $(a, b)$ in the $T C$-th iteration; and $C_{H}^{b s}$ is the cost of the control vehicles corresponding to the route of the best-sofar ant of the VRP when the number of the bases is $H$.

\subsection{Steps of the algorithm}

Step 1: Input $M$ and $N$ (the numbers of base location alternatives and control demand points, respectively), $t_{\max }$ (maximum travel time of a control vehicle), and the distance matrix between the points. Set $K_{\max }$ (a large enough number) as the ceiling number of control vehicles, $t c_{1}$ as the time of the search by the ants in the upper level and $t c_{2}$ as the time of the search by the ants in the lower level.

Step 2: Set $H=1, t c_{1}=1, C_{\text {min }}$ as a large enough number, and calculate the fixed $\operatorname{cost} c_{H}$.

Step 3: Solve the FLP in the upper level by the ant colony algorithm.

Step 3.1: Generate $M$ ants in the upper level. The initial pheromones between alternative check bases are calculated by Equation 20. The ants' expectations of selecting other alternative check bases from the current one are calculated by Equation 12.

Step 3.2: Each ant randomly selects an alternative check base to start generating the route, and $p_{a b}(t)$ is calculated by Equation 13. To avoid falling into a local optimization, the roulette selection method is used to increase the randomness of the selection [39]. The details are: generating a random number $\varepsilon, \varepsilon \in(0,1)$ and successively accumulating the $p_{a b}(t)$ according to the ID of the feasible alternative check bases. When $\sum p_{a b}(t) \geq \varepsilon$, the alternative check base with the largest ID code is selected. Then, the local pheromone updating is according to Equations 19 and 20. After all ants in the upper level complete their location selection, go to Step 3.3.

Step 3.3: Number the routes of ants in the upper level as $1,2,3, \ldots, M$. Let $y=1$ denote the first route. 
Step 4: Group the nodes in route y to form a node set and map the set to a virtual node; in this case the ants in the lower level will use this virtual point as the starting point for route generation.

Step 4.1: Generate $\mathrm{N}$ ants in the lower level. The initial pheromones between each pair of demand points are calculated by Equation 16, and the initial pheromones between each demand point and the virtual node is equal to the initial one between the demand point and the corresponding nearest check base.

Step 4.2: The ant starts from the virtual node and selects the next demand point based on Equation 14 and the roulette selection method first. Then, determine the check base o where the ant will return by the route selection method in Section 2.2.

Step 4.3: To ensure the cruise time $t$ is smaller than $t_{\max }$, it is necessary to judge whether to add the selected demand point to the ant's travel route. The method is as follows: set $j$ as the selected demand point, calculate $t$ if $j$ is added to the travel route and time $\left(t_{j o}\right)$ of the ant from $j$ to $o$. If $t+t_{j o} \leq t_{\max }$, then add $j$ to the route. The local pheromone is updated by Equations 15 and 16 and then continue to run Step 4.3. If $t+t_{j o}>t_{\max }$, then $j$ is not added to the route and the current node is the last visited demand point. Let the ant return to $o$ and set $t=0$ to run Step 4.2-Step 4.3 again.
Step 4.4: After all the ants in the lower level finish a round of searching, the global pheromone is first updated by Equations 17 and 18. Then, calculate the variable $\operatorname{cost} c_{V}$ (including fuel cost, vehicle purchase cost, etc.) corresponding to the optimal route in the current round of the search. Update the cost for the overloaded truck control as $C=c_{V}+c_{H}$; if $C<C_{\text {min }}$, set $C_{\text {min }}=C$ and $t c_{2}=t c_{2}+1$, and then run Step 4.1-Step 4.4 again. If $t c_{2}>t c_{2}^{\max }$, set $y=y+1$ and run Step 4-Step 4.4 again. If $y>M$, go to Step 5.

Step 5: Update the global pheromone by Equations 21 and 22 , set $t c_{1}=t c_{1}+1$ and then run Step 3.1-Step 5 again. If $t c_{1}>t c_{1}^{\max }$, run Step 6.

Step 6: Set $H=H+1$ and calculate $c_{H}$. If $c_{H}<C_{\min }$, set $t c_{1}=t c_{2}=1$ and run Step 3-Step 6 again. Otherwise, stop the calculation and output the global optimal solution.

\section{CASE ANALYSIS}

A survey from the project of "Guiyang rural highway maintenance pilot plan" revealed that 63 roadways have seriously overloaded trucks, including X079, X153, X192, X072, X063, X184, X074, $\mathrm{X} 191, \mathrm{X} 124$ and X071. The data are picked up from our survey in 2017 which is a part of one of our projects. The names of 63 roadways with seriously overloaded trucks in Guiyang rural highway network are shown in Table 1.

Table 1 - Names of the 63 roadways

\begin{tabular}{||c|c|c|c|c|c|c|c||}
\hline ID & Roadway & ID & Roadway & ID & Roadway & ID & Roadway \\
\hline \hline 1 & X166 & 17 & S308 & 33 & X129 & 49 & S310 \\
\hline 2 & S420 & 18 & S211 & 34 & S102 & 50 & S102 \\
\hline 3 & Y020 & 19 & X195 & 35 & S101 & 51 & S210 \\
\hline 4 & Y003 & 20 & X073 & 36 & X164 & 52 & S210 \\
\hline 5 & S310 & 21 & Y049 & 37 & S207 & 53 & S421 \\
\hline 6 & X163 & 22 & S106 & 38 & X153 & 54 & S210 \\
\hline 7 & X165 & 23 & X067 & 39 & S420 & 55 & X078 \\
\hline 8 & S208 & 24 & S209 & 40 & Y066 & 56 & S104 \\
\hline 9 & S310 & 25 & Y042 & 41 & X184 & 57 & Y071 \\
\hline 10 & Y019 & 26 & S210 & 42 & S101 & 58 & X127 \\
\hline 11 & Y014 & 27 & S210 & 43 & Y004 & 59 & S105 \\
\hline 12 & Y024 & 28 & X178 & 44 & Y012 & 60 & S210 \\
\hline 13 & S310 & 29 & S209 & 45 & Y024 & 61 & X065 \\
\hline 14 & Y005 & 30 & Y014 & 46 & S209 & 62 & X114 \\
\hline 15 & S211 & 31 & X170 & 47 & X089 & 63 & S310 \\
\hline 16 & S209 & 32 & S308 & 48 & X122 & &
\end{tabular}




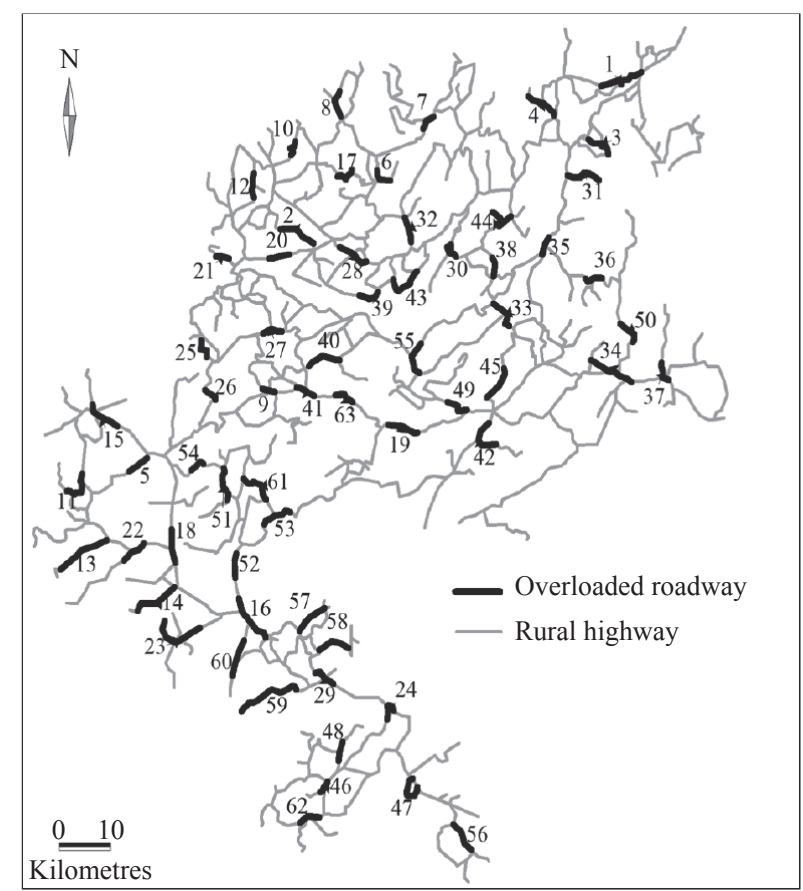

Figure 3 - Distribution of 63 roadways

The spatial distribution of 63 roadways is shown in Figure 3.

For the case study, first the midpoints of 63 roadways are set, which have the heaviest overloaded truck traffic in Guiyang rural highway, as demand points being visited by control vehicles and location alternatives of the check bases. And then, Dijkstra algorithm is used to calculate the shortest travel distance between the demand points. In this way, the studying issue has become a location-VRP problem, namely, determination of the loops for dispatching control vehicles to visit each site (named demand point) and the sites for setting the check bases. The spatial distribution and distance matrix of the demand points is shown in Figure 4.

It is assumed that the maximum number of control vehicles is 63 , a control vehicle needs three staff members and the travel speed is $40 \mathrm{~km} / \mathrm{h}$. The working time in a demand point is 0.5 hours, and the maximum cruise time is five hours. The fixed cost of constructing a check base is 2.0 million yuan. The depreciation cost of a check base is 0.2 million yuan/year with 10 years of service life. The labour cost of a staff is 60,000 yuan/year/person. The purchase cost of a control vehicle is 0.1 million yuan, and its service life is 10 years. The vehicle depreciation cost is 10,000 yuan/year. The fuel cost for vehicle travel is 120 yuan $/ \mathrm{km} /$ year if a

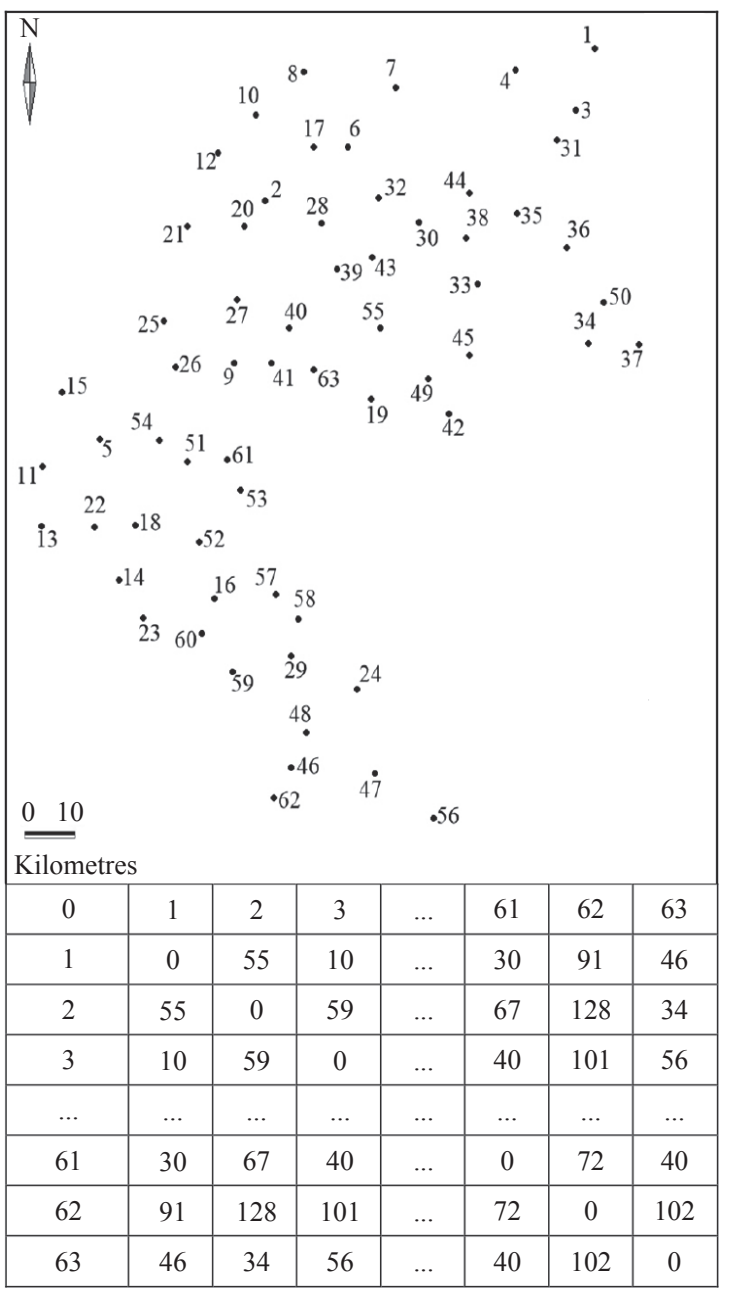

Figure 4-Spatial distribution and distance matrix of demand points

vehicle needs to check overloaded trucks 120 times in one year (the unit fuel cost of control vehicles is 1 yuan $/ \mathrm{km})$.

The solution algorithm program is compiled by Excel 2016-VBA. Setting the number of ants both in the upper level and the lower level as 63 , the computation iterations as 100 , the heuristic factors of pheromone concentration as 1 , and the heuristic factors of expectation as 2, all the pheromone evaporation coefficients as 0.5 , the sum of the fixed construction cost, the purchase cost of control vehicles and staff labour cost $\left(c_{H}=20 H+H+3 \cdot 6 H=38 H /\right.$ year, $H$ is the number of bases being built) can be obtained in the case that at least one vehicle must be purchased for each check base.

When $H=9, c_{9}$ is greater than $C_{2}, C_{3}, C_{4}$ and $C_{5}$, which means that the minimum total cost for controlling the overloaded trucks has been generated in $C_{2}, C_{3}, C_{4}$ and $C_{5}$, namely, the optimal 
location-routing scheme has been found. Therefore, we stop the solution calculation and output the schemes with the minimum total cost.

Figure 5 shows the change of the increment in ant pheromone as the increment of the calculation iterations when the number of built check base increases from 1 to 8 . The ordinate is the average pheromone increment of the 63 ants, and the abscissa denotes the number of iterations.

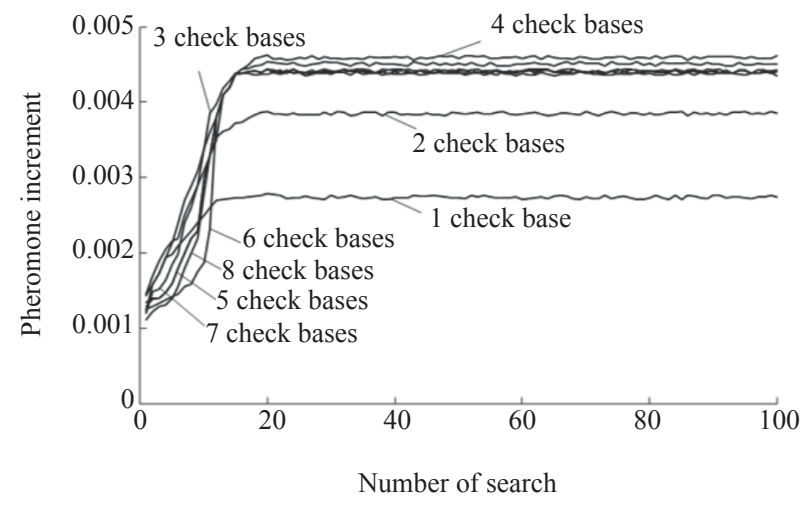

Figure 5 - Relationship between the pheromone increment and the iteration number in the route optimization

It can be seen from Figure 5 that the pheromone increments in the cases of different base numbers increase at the initial stage of the calculation. However, the increase rate gradually decreases until convergence, which indicates that there is minimum overloaded truck control cost for different numbers of check bases. When four check bases are set, the convergent value of the pheromone increment is the largest among the eight cases, which means the case with the smallest control cost.

Table 2 shows the location combination, number of control vehicles and total cost for overloaded trucks control corresponding to the cases of 1-8 check base(s) being set.

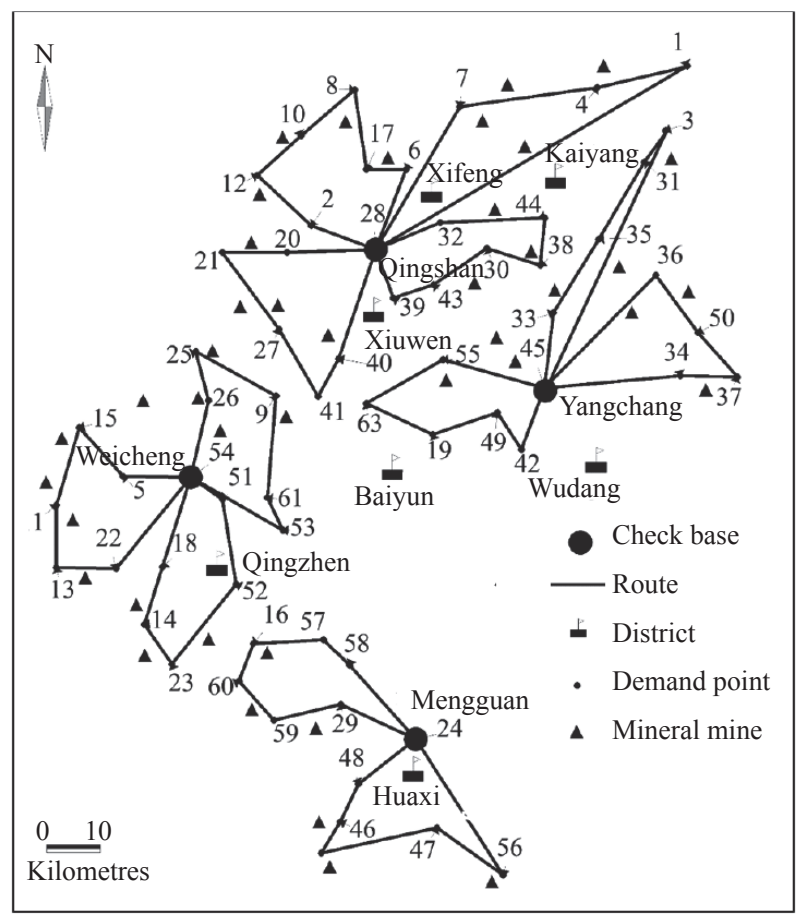

Figure 6-Scheme of the location and VRS

As seen in Table 2, when four check bases are built, they will be located at points $24,28,45$, and 54. Additionally, twelve control vehicles should be equipped, and three vehicles each are allocated to Point 45 and Point 54, two vehicles are allocated to Point 24, and four vehicles are given to Point 28 . The cruise routes of the vehicles are shown in Figure 6.

The check bases No. 24, 28, 45, and 54 are located in Mengguan Township of Huaxi District, Qingshan Township of Xifeng County, Yangchang Town of Wudang District, Guiyang and Weicheng Town of Qingzhen City, respectively. The areas around these bases are rich in mineral mines and construction sites, and the mineral trucks, slag trucks, and other large trucks in these areas are often overloaded.

Table 2 - Indicators in the cases of different numbers of check bases

\begin{tabular}{||c|c|c|c|c||}
\hline $\begin{array}{c}\text { Number of } \\
\text { bases }\end{array}$ & Total cost & $\begin{array}{c}\text { Location } \\
\text { combination }\end{array}$ & $\begin{array}{c}\text { Number of } \\
\text { vehicles }\end{array}$ & Cruise time \\
\hline \hline 1 & 450.140 & 54 & 21 & 96.375 \\
\hline 2 & 342.388 & 16,28 & 15 & 67.725 \\
\hline 3 & 321.424 & $16,28,35$ & 13 & 61.550 \\
\hline 4 & 320.192 & $24,28,45,54$ & 12 & 56.900 \\
\hline 5 & 338.944 & $16,24,28,35,40$ & 12 & 54.300 \\
\hline 6 & 358.644 & $7,24,28,35,40,54$ & 12 & 53.675 \\
\hline 7 & 378.056 & $7,16,24,28,35,40,45$ & 12 & 52.450 \\
\hline 8 & 397.509 & $7,16,24,28,35,40,45,54$ & 12 & 51.310 \\
\hline
\end{tabular}




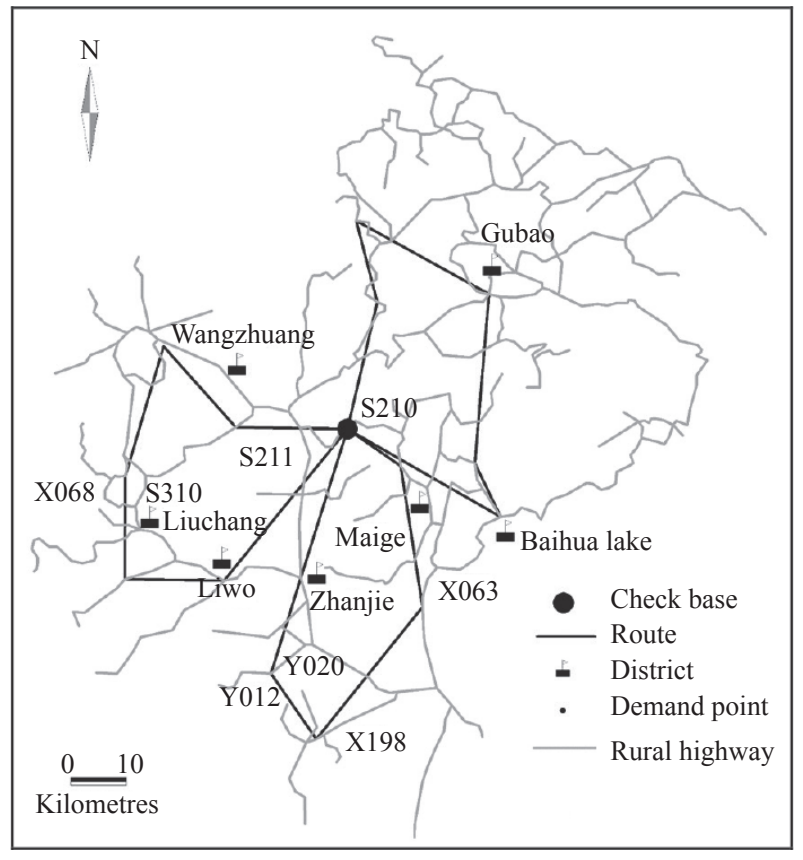

Figure 7 - Situation along the routes

The check bases can manage the inspection of the overloaded trucks, which may also act as a deterrent to the trucks in the roadways in the nearby regions.

By taking No. 54 check base as an example, the situation along the cruise routes can be seen from Figure 7. It can be known that the cruise routes of the three control vehicles pass through nine towns (Liwo Town, Liuchang Town, Wangzhuang Town, Weicheng Town, Zhanjie Town, Gubao Town, Maige Town, Baihua lake Town, Qingzhen City), three scenic spots such as Hongfeng Lake, as well as several coal mines. The main involved roadways are four provincial highways (S310, S106, S211 and S210), and many county-level and township-level rural highways, such as X068, X198, X063, Y019, Y012, Y020 and Y021. There are rich mines along the roadways, and the mining trucks tend to be overloaded.

The total cruising time means the sum of the cruising time of all control vehicles. When ensuring all demand points be visited, the smaller it is the higher the control working efficiency. The ratio of the number of demand points to the total cruising time (Equation 23) can be used to evaluate this efficiency. The idle ratio refers to the rate of the unused vehicles to the total (Equation 24). The smaller it is, the higher the utilization of the vehicles.

$W=\frac{N}{\sum_{k \in K} t_{k}}$ where: $W$ is the working efficiency of the control vehicles; $N$ is number of demand points; $t_{k}$ is the cruising time of vehicle $k$; $K$ is the control vehicle set.

$I R=\left(1-\frac{T}{K_{n} \cdot t_{\max }}\right) \cdot 100 \%$

where: $I R$ is the idle ratio of vehicles; $K_{n}$ is the number of vehicles; $t_{\max }$ is the maximum cruising time of a vehicle; $T$ is the total cruising time of all vehicles.

When 1-8 check bases are built, the working efficiency and idle ratio of the control vehicles in the optimal scheme are shown in Figure 8. It can be seen that the vehicle working efficiency increases as the increment of check bases, while the increasing trend gradually flattens out. When the number of check bases increases from one to four, the working efficiency increases significantly. This means that the working efficiency increases obviously as the increment of check bases when a few check bases are set. When the number of check bases increases from four to eight, the control efficiency does not change significantly. At this time, although there are more bases, the vehicle working efficiency does not change significantly. In contrast to the working efficiency, the idle ratio of vehicles shows a downward trend as the increment of the check bases increases from one to four, and the idle ratio is $5.1 \%$ when there are four bases, which is the lowest ratio. However, when the bases increase from four to eight, the idle ratio of vehicles increases obviously.

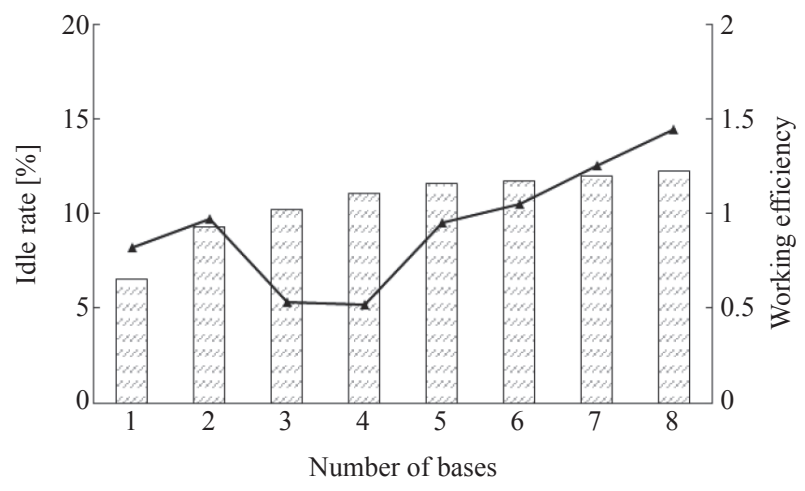

Working efficiency $\leftarrow$ Idle rate

Figure 8-Working efficiency and idle rate of vehicles under different numbers of bases

The performance of cost for controlling overloaded trucks is used to describe the relationship between the control cost and the working efficiency. The larger it is, the better the cost performance will be. The calculation method is as Equation 25.

$C P=\frac{W}{C}$ 


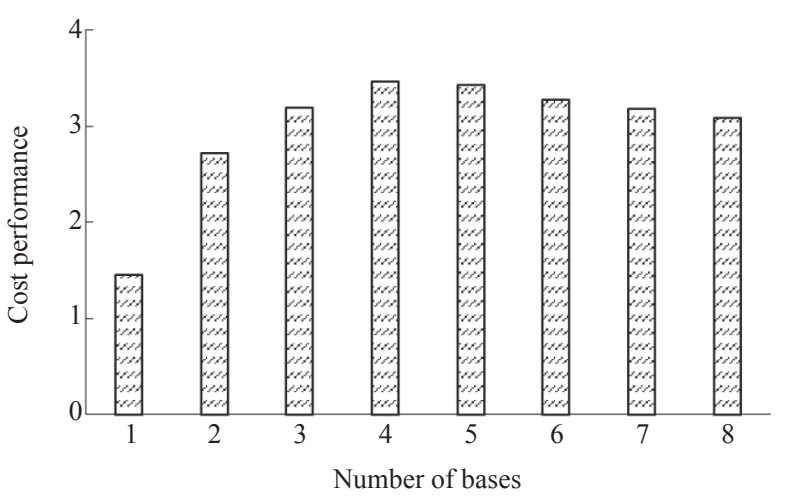

Figure 9-Cost performance for overload control under different numbers of bases

where: $C P$ is the cost performance; $W$ is working efficiency; and $C$ is the total cost for controlling overloaded trucks (unit: 10 million).

In the cases of setting 1-8 check bases, the cost performances are shown in Figure 9. It can be seen that as the number of bases increases from one to four, the cost performance increases significantly. In the case of four bases, the cost performance is 3.458 , which is the best. The cost performance shows a downward trend as the bases increase from four to eight.

It can be seen that when the check bases are located at Points 24, 28, 45 and 54, all demand points can be visited in the cruising scheme in Figure 6, and the control cost is the lowest. At this time, the base locations, the allocated control vehicles, and the cruising routes are the best ones. As seen from Figures 8 and 9, the idle ratio of control vehicles is the lowest, and the performance of the cost for controlling the overloaded trucks is the best in the optimal scheme.

\section{CONCLUSION}

In the context of the control of overloaded trucks by mobile facilities based on check bases for rural highways, a programming model is established to locate the check bases and find the routes for control vehicles. Under the condition that the set size of location alternatives is large and the number of settable check bases unknown, a multiple ant colony optimization algorithm is designed to solve the model.

Based on the actual data of the Guiyang rural highway system, it is shown that by the optimal scheme of base location and control vehicle routing, the idle ratio of control vehicles is only $5.1 \%$, which is the lowest level. In addition, the performance of cost for controlling the overloaded trucks is also the best (3.458). These results show that the proposed model and the corresponding algorithm can effectively help decision-makers to make a reasonable decision on the location of check bases and the routing of control vehicles.

In this paper, the temporal distribution of the traffic flow at demand points is not considered; thus, the optimized schemes cannot tell the departure times of the control vehicles. In our follow-up study on this topic, we will consider the traffic flow fluctuation on roadways crossing several time windows when optimizing the cruising routes and the departure times for control vehicles. In this way, we may enable the control vehicles to intercept more vehicles during the cruising.

\section{ACKNOWLEDGEMENT}

This research was supported by the Science and Technology Project of the Department of Transport, Guizhou Province (2018-121-027).

蒋劲羽 博士研究生 ${ }^{1}$

电子邮箱: jiangjy@dlmu.edu.cn 赵旭 博士 1

电子邮箱: zhao xu@126.com

郭威佑 博士研究生 ${ }^{1}$

电子邮箱: 18840839938@163.com

杨忠振（通讯作者）博士 1,2

电子邮箱: yangzhongzhen@nbu.edu.cn

1 大连海事大学交通运输工程学院

2 宁波市宁波大学海运学院

\section{农村公路超载货车的控制方案}

本文首先提出了一种新的农村公路超载治理模 式, 并建立选址一路径模型优化治超站的分布和治 超车的路径方案。其次, 针对具有大规模候选位置 集和治超站数量未知的选址-路径模型, 设计了一种 多蚁群优化算法进行求解。最后, 利用贵阳市农村 公路的实际数据进行了数值分析。研究结果表明, 该模型可以获得最优的治超站位置和治超车路径方 案, 从而验证了模型和算法的可行性, 可以帮助管 理人员做出确定治超站选址和治超车路径选择的决 策。

\section{关键词:}

公路交通; 选址一路径模型; 多蚁群

优化算法；治超站；农村公路

\section{REFERENCES}

[1] Zeng FQ, Huang XM. Asphalt pavement stress under overloading. Journal of Traffic and Transportation Engineering. 2004;4(3): 8-10.

[2] Li L, Wu QQ. Research on countermeasures of longterm oversize and overload controlling base on relational 
Jiang J, Zhao X, Guo W, Yang Z. Scheme of Overloaded Truck Control on a Rural Highway

interest factors. Journal of Highway and Transportation Research and Development. 2008;25(3): 153-158.

[3] Bagui, Das A, Bapanapalli C. Controlling Vehicle Overloading in BOT Projects. Procedia - Social and Behavioral Sciences. 2013;104(2): 962-971.

[4] Moreno-Quintero E, Fowkes T, Watling D. Modelling planner-carrier interactions in road freight transport: Optimisation of road maintenance costs via overloading control. Transportation Research Part E: Logistics \& Transportation Review. 2013;50(1): 68-83.

[5] Li ZK, Rong ZH. Research on long term solution mechanism and countermeasure to overload and oversize transportation of highway. China Journal of Highway and Transport. 2005;18(4): 96-99.

[6] Chen YS. Long-term effective solution to the overload transportation with economic lever. China Journal of Highway and Transport. 2004;17(02): 95-100.

[7] Salhi S, Rand GK. The effect of ignoring routes when locating depots. European Journal of Operational Research. 1989;39(2): 150-156.

[8] Nagy G, Salhi S. Location-routing: Issues, models and methods. European Journal of Operational Research. 2007;177(2): 649-72.

[9] Luo YB, Sun YM. Capacitated Location routing problem based on fuzzy time windows. Systems Engineering. 2014;32(01): 19-25.

[10] Nadizadeh A, Hosseini Nasab H. Solving the dynamic capacitated location-routing problem with fuzzy demands by hybrid heuristic algorithm. European Journal of Operational Research. 2014;238(2): 458-70.

[11] Cheng XQ, Jia JT, Li Y, Liu XW. Bi-level programming and algorithm on the location of arterial highway management station. Journal of Transportation Systems Engineering and Information Technology. 2016;16(3): 207-213.

[12] Caballero R, Mercedes González, Guerrero FM, et al. Solving a multiobjective location routing problem with a metaheuristic based on tabu search. Application to a real case in Andalusia. European Journal of Operational Research. 2007;177(3): 1751-63.

[13] Zhao J, Verter V. A bi-objective model for the used oil location-routing problem. Computers \& Operations Research . 2014; 62.

[14] Govindan K, Jafarian A, Khodaverdi R, Devika K. Two-echelon multiple-vehicle location-routing problem with time windows for optimization of sustainable supply chain network of perishable food. International Journal of Production Economics. 2014;152(2): 9-28.

[15] Sun QW, Zhang Y. Study on Location-routing Problem with Simultaneous Pickup and Delivery. Journal of Transportation Engineering and Information. 2017;15(2): 100-104.

[16] Laporte G, Nobert Y. An exact algorithm for minimizing routing and operating costs in depot location. European Journal of Operational Research. 1981;6(2): 224-226.

[17] Laporte G, Nobert Y, Taillefer S. Solving a Family of Multi-Depot Vehicle Routing and Location-Routing Problems. Transportation Science. 1988;22(3): 161-172.

[18] Akca Z, Berger RT, Ralphs TK. A branch-and-price algorithm for combined location and routing problems under capacity restrictions. Operations Research/
Computer Science Interfaces. 2009;47: 309-330.

[19] Srivastava R. Alternate solution procedures for the location-routing problem. Omega. 1993;21(4): 497-506.

[20] Murty KG, Djang PA. The U.S. Army National Guard's Mobile Training Simulators Location and Routing Problem. Operations Research. 1999;47(2):175-182.

[21] Balakrishnan A, Ward JE, Wong RT. Integrated facility location and vehicle routing models: Recent work and future prospects. American Journal of Mathematical and Management Sciences. 1987;7(1-2): 35-61.

[22] Salhi S, Nagy G. Consistency and robustness in location-routing. Studies in Locational Analysis. 1999;13(3): 3-19.

[23] Srivastava R, Benton WC. The location-routing problem: Considerations in physical distribution system design. Computers \& Operations Research. 1990;17(5):427-435.

[24] Barreto S, Ferreira C, Paixão J, Santos BS. Using clustering analysis in a capacitated location-routing problem. European Journal of Operational Research. 2007;179(3): 968-977.

[25] Wu TH, Low C, Bai JW. Heuristic solutions to multi-depot location-routing problems. Computers \& Operations Research. 2002;29(10): 1393-1415.

[26] Salhi GN. Nested heuristic methods for the location-routeing problem. The Journal of the Operational Research Society. 1996;47(9): 1166-1174.

[27] Nambiar JM, Gelders LF, Wassenhove LNV. A large scale location-allocation problem in the natural rubber industry. European Journal of Operational Research. 1981;6(2): 183-189.

[28] Albareda-Sambola M, Díaz JA, Fernández E. A compact model and tight bounds for a combined location-routing problem. Computers \& Operations Research. 2005;32(3): 407-428.

[29] Bouhafs L, Hajjam A, Koukam A. A Combination of Simulated Annealing and Ant Colony System for the Capacitated Location-Routing Problem. Knowledge-based Intelligent Information \& Engineering Systems, International Conference, Kes, Bournemouth, Uk, October, Part I. Springer-Verlag; 2006.

[30] Marinakis Y, Marinaki M. A Bilevel Genetic Algorithm for a real life location routing problem. International Journal of Logistics Research \& Applications. 2008;11(1): 49-65.

[31] Yu B, Jin PH, Yang ZZ. Two-stage heuristic algorithm for multi-depot vehicle routing problem with time windows. Systems Engineering - Theory \& Practice. 2012;32(8): 1793-1800.

[32] Dorigo M, Maniezzo V, Colorni A. Ant system: Optimization by a colony of cooperating agents. IEEE Transactions on Systems Man \& Cybernetics Part B Cybernetics. 1996;26(1): 29-41.

[33] Yao BZ, Hu P, Zhang MH, Tian XM. Improved ant colony optimization for seafood product delivery routing problem. Promet - Traffic\&Transportation. 2014;26(1): 1-10.

[34] Yu B, Yang ZZ, Yao BZ. An improved ant colony optimization for vehicle routing problem. European Journal of Operational Research. 2009;196(1): 171-176.

[35] Gambardella LM, Taillard R, Agazzi G. MACS-VRPTW: A Multiple Ant Colony System for Vehicle Routing 
Problems with Time Windows. New Ideas in Optimization. 1999; 63-76.

[36] Ting CJ, Chen CH. A multiple ant colony optimization algorithm for the capacitated location routing problem. International Journal of Production Economics. 2013;141(1): 34-44.

[37] Ma JH, Fang Y, Yuan J. Mutation ant colony algorithm for multiple-depot multiple-types vehicle routing prob- lems with shortest finish time. Systems Engineering Theory and Practice. 2011;31(8): 1508-1516.

[38] Dorigo M, Stützle T. Ant Colony Optimization. IEEE; 2004.

[39] He YZ, Yang ZZ. Optimization of express distribution by cooperatively using private trucks and buses. Journal of Traffic and Transportation Engineering. 2017;17(6): 97-103. 